KLEINE TEXTE FÜR VORLESUNGEN UND ÜBUNGEN HERAUSGEGEBEN VON HANS LIETZMANN

66

\title{
DIE \\ FRÖSCHE DES ARISTOPHANES
}

MIT AUSGEWÄHLTEN ANTIKEN SCHOLIEN

HERAUSGEGEBEN

vON

WILHELM SÜSS

UNVERÄNDERTER NEUDRUCK

BERLIN

VERLAG VON WALTER DE GRUYTER \& CO.

1935 
Arehiv-Nr. 333235 